\title{
Efficacy of The Acupressure Wrist-Ankle Strap Combined With CBTI In Mild Insomnia Patients With Anxiety Disorders: Study Protocol For A Randomized Controlled Trial
}

\author{
Ying Yuan ( $\nabla$ m14782395536@163.com ) \\ Changhai Hospital https://orcid.org/0000-0002-6155-1303 \\ Qinghui Zhou \\ Changhai Hospital \\ Fanfu Fang \\ Changhai Hospital \\ Weihong Li \\ Changhai Hospital \\ Yanli You \\ Changhai Hospital
}

\section{Research Article}

Keywords: anxiety, comorbid insomnia, acupressure wrist-ankle strap, HPA axis, RCT, study protocol

Posted Date: May 26th, 2021

DOI: https://doi.org/10.21203/rs.3.rs-414035/v1

License: (c) (1) This work is licensed under a Creative Commons Attribution 4.0 International License.

Read Full License 


\section{Abstract}

Background: Insomnia is very common in current society, and patients are often accompanied by a certain degree of anxiety, depression, etc. Recent studies have found that the hypothalamic-pituitaryadrenal (HPA) axis excitement-inhibition state is an important indicator of sleep quality. Wrist-ankle acupuncture (WAA) is safe and effective for insomnia. The acupressure wrist-ankle straps, based on WAA theory, is a portable WAA point compression strap, which can treat diseases by automatically applying pressure to the treatment location and be operated by patients themselves. We design this trial to evaluate the clinical effect of the acupressure wrist-ankle strap in the treatment of mild insomnia patients with anxiety disorders.

Methods/design: This trial is a parallel-design, patients-assessor blinded, randomized, sham-controlled. In total, 108 patients diagnosed with mild insomnia patients with anxiety disorders will be randomly equally assigned into two groups, acupressure wrist-ankle strap group or non-acupressure wrist-ankle strap group; they will receive treatments for 8 weeks with 5 sessions each week. Rating scales, sleep monitor and laboratory tests will be used to observe the clinical effect. From the perspective of the circadian secretion of peripheral blood-related hormones in the hypothalamic- pituitary- adrenal (HPA) axis, the possible mechanism of acupressure wrist-ankle straps for treating insomnia is studied.

Discussion: The results of this study will confirm the efficacy of acupressure wrist-ankle strap combined with CBTI in the treatment of mild insomnia patients with anxiety disorder and whether its mechanism is related to HPA axis. The acupressure wrist-ankle strap may become a pure physical, no side effect treatment of mild insomnia.

Trial registration: Chinese Clinical Trial Registry (identifier: ChiCTR2000039352; registration date: 24 October 2020).

\section{Background}

After a lapse of 9 years, the British Psychopharmacological Association (BAP) updated its consensus on the treatment of insomnia, parasomnia, and circadian rhythm disorders in 2019. The consensus mentioned that in all patients with diagnosed insomnia, about half of them have at least one comorbidity of mental disorder ${ }^{1}$. Insomnia patients are often accompanied by a certain degree of anxiety, depression and other bad moods. The specific manifestations of insomnia patients with anxiety disorder include insomnia with significant anxiety symptoms, or insomnia with emotional disorders such as nervousness, worry and irritability ${ }^{2}$. The survey found that about $50 \%$ of insomnia patients are accompanied by mental disorders ${ }^{3}$. Compared with non-anxiety insomnia patients, insomnia patients with anxiety have significantly reduced sleep efficiency, total sleep time, and percentage of slow-wave sleep, which significantly reduces the quality of life of patients and brings serious social dysfunction and economic burden ${ }^{45}$. 
Although the pathogenesis of insomnia has not yet been fully understood, studies ${ }^{6}$ have found that the excitation-inhibition state of the hypothalamic-pituitary-adrenal (HPA) axis, is an important indicator of sleep quality. The HPA axis is mainly composed of the hypothalamus, pituitary gland and adrenal glands, and its activity is regulated by corticotrophin releasing hormone (CRH). CRH promotes the secretion of adreno-cortico-tropic-hormone (ACTH) from the pituitary. ACTH is released into the blood and reaches the adrenal gland through systemic circulation, prompting the adrenal cortex to release glucocorticoids and cortisol (CORT). ACTH can improve the irritability of the cerebral cortex and is an important part of the sleep-wake regulation mechanism. Many monoamine neurotransmitters, such as serotonin $(5-\mathrm{HT})$ and norepinephrine (NE), also play an important role in the regulation of the HPA axis ${ }^{7}$.

It's vital to treat insomnia. At present, antidepressants and benzodiazepines with sedative effects, as well as new types of hypnotics, are often used for patients with anxiety and insomnia. However, patients may have dizziness, drowsiness, cognitive dysfunction, apnea and other adverse reactions after taking such drugs $^{8}$. In addition to their own adverse drug reactions, there were also insomnia rebound and withdrawal reactions. Patients with mild insomnia have occasional insomnia, which often does not affect work or has little effect, and often does not require drug treatment. It can be improved by non-drug treatment represented by sleep hygiene education and cognitive behavioral therapy for insomnia $(\mathrm{CBTI})^{9}$, and most of them are against medication. Therefore, it is of great significance for patients with mild insomnia to develop a purely physical, easy-to-operate, non-invasive, non-toxic and side-effect and repeated use of drug replacement therapy.

The acupressure wrist-ankle strap is a device developed on the basis of the WAA therapy with a history of more than 50 years in China. The therapy is pure physical therapy, non-invasive, painless, non-toxic and side-effects, environmentally friendly, low-carbon, recyclable, adjustable by individual, and easy to operate. Our clinical experience indicates that patients with mild insomnia can quickly calm down and enter sleep state after using the strap. Clinical studies have shown that acupressure wrist-ankle strap has a good effect on insomnia ${ }^{1011}$. However, there is no clinical randomized controlled study to prove the efficacy for insomnia. We are trying to find a more effective physical therapy for insomnia by using CBTI combined with acupressure wrist-ankle strap to treat mild insomnia with anxiety disorder.

\section{Methods/design}

\section{Study design}

This is a two-armed, double-blind, randomized, sham-controlled clinical trial aiming to explore the efficacy of the acupressure wrist-ankle strap for insomnia patients with anxiety disorder.

A total of 108 participants, from the department of acupuncture and moxibustion and the department of internal medicine of traditional Chinese medicine of Shanghai Changhai Hospital, diagnosed with mild insomnia and anxiety disorder in accordance with Guidelines for the Diagnosis and Treatment of Insomnia in China formulated by the Chinese Sleep Medicine Congress (CSMC, 2017 revised version)3 
and Expert Consensus on Diagnosis and Treatment of Anxiety and Depression in General Hospitals formulated by Anxiety disorder cooperation group, Psychiatry Branch, Chinese Medical Association (2012 revised version $)^{12}$ will be randomly assigned to two equal groups with 54 patients in each group, including acupressure and non-acupressure wrist-ankle strap group. The outcomes include scales, objective parameters detected by device (wActiSleep-BT Actigraph equipment) and laboratory tests, which will be used to observe the efficacy of the strap on insomnia patients and study its possible mechanism of circadian secretion of related hormones in peripheral blood of hypothalamic-pituitaryadrenal axis (HPA axis). Our study will last 13 weeks in total. The protocol has been registered on Chinese Clinical Trial Registry (identifier: ChiCTR2000039352) and will be conducted following Helsinki declaration. This trial will be reported according to the Consolidated Standards of Reporting Trials (CONSORT) statement ${ }^{13}$. Figure 1 is the flowchart of this study.

The study flow-chart is summarized in Fig. 1.

\section{Participants}

Patients meeting the diagnosis of Guidelines for the Diagnosis and Treatment of Insomnia in China formulated by CSMC in 2017 and Expert Consensus on Diagnosis and Treatment of Anxiety and Depression in General Hospitals formulated by Anxiety disorder cooperation group, Psychiatry Branch, CMA in 2012 will be recruited. Among them, insomnia is the main clinical manifestation of these patients, and the main complaints are difficulty falling asleep, difficulty maintaining sleep, waking up early, refusing to go to bed at a suitable time, difficulty falling asleep without nursing care, and accompanied by daytime fatigue, irritability and the decline of work and social ability. It occurs at least 3 times a week and lasts for more than 1 month. Patients with mild insomnia mainly manifest as occasional insomnia (3 times $\leq$ attacks per week $<5$ times), often wake up or sleep instability during sleep, and wake up early in the morning, but it has no or minimal impact on the job; PSQI score is $7 \llbracket 11$ points. And exclude secondary insomnia caused by various diseases and other factors.

\section{Inclusion criteria}

Eligibility for participation requires each of the following criteria be met:

- Symptoms comply with both the Guidelines for the Diagnosis and Treatment of Insomnia in China formulated by the CSMC in 2017 and Expert Consensus on Diagnosis and Treatment of Anxiety and Depression in General Hospitals formulated by Anxiety disorder cooperation group, Psychiatry Branch, CMA in 2012

- The occurrence of insomnia was 3 times (including) to 5 times per week.

- The Pittsburgh Sleep Quality Index (PSQI) score was 7 to 11, and the Hamilton Anxiety Scale (HAMA) score> 14.

- Age 18-70 years at the time of enrollment (either sex).

- Haven't received WAA or other similar treatments. 
- Unwilling to use any drugs.

- Signed informed consent.

\section{Exclusion criteria}

Patients who meet any of the following criteria will be excluded:

- Suffering from insomnia caused by mental diseases or drugs.

- Dependence or abuse of alcohol or other substances.

- Currently pregnant or lactating.

- Having any serious disease of cardiovascular, liver, kidney, hematopoietic system, and mental patients.

- Allergic to the material of the wrist-ankle straps.

- Have taken sedative and hypnotic drugs within 1 month.

\section{Dropout criteria}

The researcher decides to withdraw from the trial:

- Patient's condition progressed or continues to deteriorate during the trial, and the clinical trial should be stopped according to the doctor's judgment.

- During the trial period, patient has severe complications or special physiological changes, and it is not suitable to continue the test.

- Serious adverse events occurred during the trial period.

- Patient has poor compliance during the trial period, and the treatment dose was less than $80 \%$ or more than $120 \%$.

Patients quit the trial by themselves:

- During the trial period, patients are unwilling to continue the treatment or take sedative and hypnotic drugs by themselves due to various reasons, and actively propose to withdraw from the clinical trial.

- Although the patients don't explicitly propose to withdraw from the trial, they might fall out naturally due to loss of follow-up.

\section{Randomization, allocation concealment}

Random sequence list is generated by using stratified block randomization design and SAS 9.4 (SAS Institute Inc., Cary, NC, USA). The sequence is stratified by gender and age. This trial has an independent statistician, assigning the eligible patients to the corresponding intervention code based on the list, who is not be involved in recruitment, implementation, data collection and data analysis of this trial. All of the random code information is kept by a certain person and stored in sealed opaque envelops. When an eligible patient is enrolled in the group, The statistician will randomized according to the above method, 
and the corresponding box within wrist-ankle strap will be given to the researcher who will be responsible for informing patients the details about the implementation, the wrist-ankle straps used in both groups will have the same packaging. The allocation concealment procedure will not be exposed until the clinical trial finished completely.

\section{Blinding}

All the patients, outcome assessors, and data analyst will be blinded to treatment allocation. Every patient of the two groups will receive the same packaging box in the above envelope after entering the group according to the allocation method, which contains the strap (acupressure wrist-ankle strap or nonacupressure wrist-ankle strap) and its instructions, which will guide patients themselves how to use the straps and informed them related precautions, including the size of the straps, tightness, selection and adjustment of compression devices and wearing time. In order to prevent the patients from communicating with each other, the form of follow-up appointments is adopted to ensure that the patients will stagger their visits and avoid communication. When the patient withdraws from the trial due to various reasons, emergency unblinding is allowed.

\section{Interventions}

The wrist-ankle acupuncture (WAA) is a unique acupuncture method. According to the principle of point selection of WAA, insomnia is a symptom that cannot be located. When doing wrist-ankle acupuncture, we choose upper 1 on both sides. In the acupressure wrist-ankle strap group, the compression component of the wrist-ankle strap compresses upper 1 to produce an acupuncture-like effect. (Figs. 2 and 3 )

The acupressure wrist-ankle strap is a device developed on the basis of WAA (Fig. 4), which is used to replace the invasive operation of WAA. The compression part of wrist-ankle strap, which is detachable, is used to apply pressure and stimulation to the corresponding WAA point (compression point) to achieve the effect of treating diseases. There is a compression component mounting base on the wrist-ankle strap, and the position can be adjusted according to the needs of the disease, and then 1 to 2 compression components can be installed. Compressing different points can alleviate the pain in different body regions, and is usually used for pain, insomnia, motion sickness, seasickness, morning sickness, etc.

\section{Acupressure wrist-ankle strap group}

This group is the intervention group. A portable WAA' point compression therapy device is used, with compression components on the inside; the bilateral upper 1 are selected as the compression points. The upper 1 is located at the depression between medial border of the ulnar and the tendon of musculus flexor carpi ulnaris, and at the level of about two fingers above the transverse crease of the wrist. A compression component is installed inside the wrist-ankle strap and worn on both wrists to ensure that the compression component can be compressed to the upper 1 compression point, as shown in Fig. 5 . 
Wear it for 30 minutes before going to bed every day, and take it off at bedtime. 2 weeks as a course of treatment, 4 courses in total. The acupressure wrist-ankle strap compression device is shown in Fig. 6 (A).

\section{Non-acupressure wrist-ankle strap group}

This group served as the control group. A portable WAA' point compression therapy device is selected. The inner compression component will automatically contract without compression, and it will be worn at the same position on both wrists. Wear it for 30 minutes before going to bed every day, and take it off at bedtime. 2 weeks as a course of treatment, 4 courses in total. The Non-acupressure wrist-ankle strap compression device is shown in Fig. 6(B).

In addition, all patients will be given sleep hygiene education and CBTI. CBTI can effectively correct the wrong sleep cognition and inappropriate behavior factors of patients with insomnia, help alleviate the difficulty of falling asleep, increase sleep time, improve sleep efficiency, improve sleep quality, eliminate psychological and physiological high wakefulness, enhance the drive to fall asleep, reconstruct correct sleep and awakening cognitive model, and can maintain long-term efficacy.

\section{Outcomes}

The time schedule of enrollment, interventions and assessments is shown in Fig. 7. The following outcomes for all the patients will be assessed in person by blinded and independent assessors.

\section{Primary outcome}

Primary outcome is the Pittsburgh sleep quality index aggregate score $\mathbb{P S Q} \mathrm{I}$, which is used to evaluate patient's general sleep quality in the past month. The PSQI consists of 19 self-assessment questions and 5 questions assessed by sleep partners. Only 19 self-assessment questions will be scored. The 19 selfassessment questions constitute 7 factors from 0 to 3 points. The cumulative score of each factor component is the total score of the PSQI. The total score ranges from 0 to 21 . Higher score means worse sleep quality ${ }^{14}$. The PSQI will be assessed at baseline, 4, 8 weeks after randomization and flow-up period.

\section{Secondary outcomes}

Pittsburgh Sleep Quality Index DPSQI The PSQI scores include seven domains, using 0, 1, 2, and 3 points to evaluate the patient's duration of sleep, sleep disturbance, sleep-onset latency, daytime dysfunction due to night sleep, sleep efficiency, need for medications to sleep, and overall sleep quality $₫$ which will be assessed at baseline, 4, 8 weeks after randomization and flow-up period.

Hamilton Anxiety Scale (HAMA) The HAMA is one of the commonly used scales in psychiatric clinics, including 14 items. As an important diagnostic tool for anxiety disorders, it is often used clinically as a basis for the diagnosis and degree classification of anxiety disorders. According to the relevant data of the scale cooperation group in China, if the score is less than 7, there is no anxiety; if the score is more than 7 , there may be anxiety; if the score is more than 14, there must be anxiety; if the score exceeds 21 
points, there must be obvious anxiety; if the total score exceeds 29 points, it may be serious anxiety. If the score of the scale is more than 14 , it can be diagnosed as anxiety disorder. HAMA will be assessed at baseline, 4, 8 weeks after randomization and flow-up period.

wActiSleep-BT monitor The Actigraphy is a small-sized wrist watch worn on the patient's wrist before going to bed, it can monitor the quality of sleep, such as the latency to fall asleep, total sleep time, number and duration of wake-ups, and sleep efficiency. The main sleep parameters are SE, total sleep time (TST) and sleep awakenings (SA). It will be assessed at baseline, 4, 8 weeks after randomization and flow-up period.

Laboratory tests The pathogenesis of insomnia is that some substances in the brain (such as neurotransmitters, hormones, etc.) act on the sleep center of the human body under the influence of various factors, leading to the abnormality of the central neurotransmitter system, and then affect sleep. There is some evidence $6^{17}$ that the pathogenesis of insomnia is closely related to the circadian secretion of peripheral blood-related hormones in the hypothalamus-pituitary-adrenal axis (HPA axis). In this study, we will detect the levels of adrenocorticotropic hormone (ACTH), cortisol (CORT), serotonin (5-HT), norepinephrine (NE) before and after treatment in the two groups.

TCM Syndrome Score Scale: Syndrome is the objective basis of syndrome differentiation and treatment in TCM. The object of this study is mild insomnia patients with anxiety disorders. So the TCM syndrome score scale includes insomnia part and depression part, the sum of the two is the total score. The syndrome scale with the characteristics of TCM theory is a necessary way to realize the standardization of TCM syndrome diagnosis and evaluation.

Except laboratory tests of all outcomes, primary outcome and other secondary outcomes are detected at baseline and every 4 weeks, laboratory data are tested at baseline and the $8^{\text {th }}$ week.

\section{Statistical analysis}

Participants will complete relevant questionnaires and laboratory tests at the first visit. Researchers will provide guidance to ensure the reliability and validity of the questionnaire; meanwhile, sleep monitor will be completed the night before the treatment.

All observation results will be documented in the clinical observation forms. Missing data will be eliminated. At the some time, a computer database will be established. The obtained data will be input into the computer on the day of observation records. SPSS 21.0 will be used for statistical processing. After normal distribution test and homogeneity test of variance are performed on measurement data, analysis of variance of repeated measurements will be used for comparison between groups, paired $t$ test will be used for comparison before and after treatment, and non-normal distribution data will be used for non-parametric test. The statistical data will be compared by $\chi 2$ test. The difference will be considered statistically significant when $\mathrm{P}<0.05$. 


\section{Sample size}

PASS 11 software (NCSS, Kaysville, UT) was used to calculate the required sample size. The sample size calculation formula as follows:

$$
n_{t}=n_{c}=\frac{\left(Z_{1-\alpha / 2}+Z_{1-\beta}\right)^{2} s^{2}(1+1 / k)}{\left(\mu_{t}-\mu_{c}\right)^{2}}
$$

Sample size calculation is based on the primary outcome, which is proposed to be the PSQI. Based on the preliminary experiments, the PSQI aggregate score of the acupressure wrist-ankle strap group was 6.125 \pm 2.93 , and the PSQI aggregate score of the control group was $8.625 \pm 3.77$. The number of cases in the two groups was 8 cases respectively. The combined standard deviation of the two groups was calculated to be 3.60. That is, in the formula, $\mu_{t}=6.125, \mu_{c}=8.625, k=1$, and $s=3.60$. In this study, $\alpha=0.05$ and $1-\beta=$ 0.9 , therefore $Z_{1-\alpha / 2}=1.96, Z_{1-\beta}=1.28 .45$ patients are required for each group. Considering an estimated $20 \%$ dropout rate, 54 patients will be enrolled in each group, giving a total of 108 patients.

\section{Trial and data monitoring}

The data will be recorded on the paper case report form by a certain assessor, and doubled-entered into the electronic case report form. Data and Safety Monitoring Committee of our hospital will monitor the data and review the progress of the trial every 3 months. Monitors will check the implementation of the study protocol, the treatment of the subjects and the completion of the informed consent documents every 3 months. The revision of the plan will be tracked and dated so that the new version can be submitted to the committee.

\section{Safety evaluation}

The acupressure wrist-ankle strap is pure physical therapy, which is safe. Patients need to pay attention not to wear it too tightly or for too long. After wearing it for about 30 minutes, take it off or loosen it to prevent pressure from causing poor local blood circulation. If the skin is itchy and rash occurs after wearing it, it may be allergic. Patients will be told to stop using the strap and be arranged for a dermatologist. If insomnia worsens after multiple treatments, the patient will be referred to the Department of Neurology for other forms of treatment.

\section{Discussion}

To the best of our knowledge, this is the first registered clinical randomized controlled study of CBTI combined with acupressure wrist-ankle strap in the treatment of insomnia. The wrist-ankle acupuncture (WAA) is a unique acupuncture method, which is mainly embodied in the theory of selecting acupuncture points according to body partitions and the subcutaneous superficial needling operation that does not achieve the de-qi sensation. According to the basic principles of WAA, each side of the body is divided 
into 6 longitudinal zones, and one point is assigned to each of the six longitudinal zones of the wrist and ankle, with the same name as the zone; each point can treat the diseases of the longitudinal zone with the same name. According to the principle of point selection of WAA, insomnia is a symptom that cannot be located. When doing wrist-ankle acupuncture, we choose upper 1 on both sides. In the acupressure wrist-ankle strap group, the compression component of the wrist-ankle strap compresses upper 1 to produce an acupuncture-like effect. The acupressure wrist-ankle strap, based on WAA theory, is a portable WAA point compression treatment strap that treats diseases by automatically applying pressure to acupuncture points, which can be operated by patients themselves. The therapy is pure physical therapy, non-invasive, painless, non-toxic and side-effects, environment-friendly and low-carbon, recyclable, and can be adjusted by people, and is easy to operate. It remains to be verified whether the acupressure wristankle strap and WAA have similarities in the treatment of insomnia. Through the study on the therapeutic effect of acupressure wrist-ankle strap, a new physical intervention method can be added for insomnia, which can be used to relieve insomnia at any time without the intervention of doctors.

In this trial, the patients with mild insomnia are often unwilling to take drugs, and there is no high risk without other treatments. Therefore, it is ethically possible for us to include a control group that receives just CBTI. This trial is a rigorously designed randomized controlled double-blind trial. The design of the non-acupressure wrist-ankle strap, the wrist-ankle strap instruction manual and follow-up appointments ensure the accuracy of the blind method of this trial. In order to ensure the quality of this research, before the research officially starts, we conducted pre-experiment first, consult experts and literature to optimize the test plan based on the pre-test situation, and submitted the study protocol to the medical ethics committee for review and approval; each stage of the trial has an independent researcher to supervise the subject and collect feedback in time; all the research participants, including researchers, receptionists and data processors, in this study will be trained to ensure the smooth implementation of the trial.

The study aims to provide evidence-based medical evidence for the prevention and treatment of insomnia by acupressure wrist-ankle strap, and promotes WAA Clinical application. The result of this trial is expected to confirm that the new type of acupressure wrist-ankle strap can significantly improve the sleep quality and anxiety disorder of patients, reduce the suffering of patients, and improve the quality of life; it can also shorten the latency to fall asleep, increase the total sleep time, reduce the number of arousals and duration, improve sleep efficiency; it can reduce ACTH, CORT, 5-HT and NA in the peripheral blood of patients with insomnia, thereby improving sleep quality.

In view of the above considerations, this study intends to sort out and summarize the clinical data of the new type of acupressure wrist-ankle strap for mild insomnia patients with anxiety disorder in this research group, and form a standardized treatment plan. A strictly designed randomized controlled study method will be used to compare the improvement of acupressure wrist-ankle strap and non-acupressure wrist-ankle strap on the patients, to further verify its efficacy and explore the possible mechanism of action, in order to enrich the treatment of insomnia, improve its clinical efficacy, and lay the foundation for the popularization and application of the new acupressure wrist-ankle strap. 
The limitation of this trial is mainly that the subjects we will observe are only patients with mild insomnia, and it is not clear whether the wrist-ankle strap is effective for patients with moderate to severe insomnia. This is because the control group is non-acupressure wrist-ankle strap, and the whole trial period is 8 weeks. Considering that the patients who used to take insomnia drugs may aggravate the disease after stopping taking the drugs, which will affect their physical and mental health, so only the patients with mild illness will be included. The efficacy of acupressure wrist-ankle strap in patients with moderate to severe insomnia will be verified in the future.

\section{Trial Status}

Participants are currently being recruited into the study. The trial began recruitment on 24 October 2020 and will be finished on 31 June 2022.

\section{Abbreviations}

BAP: the British Psychopharmacological Association; WAA: Wrist-Ankle Acupuncture; HPA: Hypothalamicpituitary-adrenal; $\mathrm{CRH}$ : Corticotrophin releasing hormone; ACTH: Adreno-cortico-tropic-hormone; CORT: Glucocorticoids and cortisol ; 5-HT: Serotonin; NE: Norepinephrine; CBTI: Cognitive Behavioral Therapy for Insomnia; CSMC: Chinese Sleep Medicine Congress; CONSORT: Consolidated Standards of Reporting Trials; PSQI: Pittsburgh Sleep Quality Index; HAMA: Hamilton Anxiety Scale;

\section{Declarations}

\section{Funding}

This trial was supported by grants from the Traditional Chinese Medicine program of Shanghai Municipal Health Commission (No. 2020LQ003), the Shanghai Science and Technology Commission medical guidance (Chinese and Western Medicine) science and technology support project (No. 19401930100).

\section{Availability of data and materials}

Not applicable.

\section{Authors' contributions}

YY participated in the conception and design of the trial, and in plans for the analysis of the data. QHZ and FFF participated in drafting the manuscript. WHL and YLY participated in data collection, and were in charge of the recruitment and treatment of patients. YY are the corresponding authors of this article. All authors discussed, read and revised the manuscript, and all approved the publication of this protocol.

\section{Ethics approval and consent to participate}


This study will be performed according to the guidance and principles of the Declaration of Helsinki. The protocol has been approved by the supervision of Shanghai Changhai Hospital Ethics Committee (ethical approval number: CHEC2020-110) and has been registered in the Chinese Clinical Trial Registry (identifier: ChiCTR2000039352). Only patients who have signed the informed consent form will be included.

\section{Consent for publication}

Not applicable.

\section{Competing interests}

The authors declare that they have no competing interests.

\section{Author details}

${ }^{1}$ Department of Traditional Chinese Medicine, Naval Medical University

Address correspondence to: Ying Yuan, Department of Traditional Chinese Medicine, Naval Medical University, No. 800 Xiangying Road, Yangpu District, Shanghai 200433, China. Email: m14782395536@163.com

\section{References}

1. Wilson S, Anderson K, Baldwin D, Dijk DJ, Espie A, Espie C, Gringras P, Krystal A, Nutt D, Selsick H, Sharpley A. British Association for Psychopharmacology consensus statement on evidence-based treatment of insomnia, parasomnias and circadian rhythm disorders: An update. J Psychopharmacol. 2019 Aug;33(8):923-947. doi: 10.1177/0269881119855343. Epub 2019 Jul 4. PMID: 31271339.

2. Sutton EL. Psychiatric disorders and sleep issues. Med Clin North Am. 2014 Sep;98(5):1123-43. doi: 10.1016/j.mcna.2014.06.009. Epub 2014 Jul 22. PMID: 25134876.

3. Zhang Peng, Li Yanpeng, Wu Huijuan, Zhao Zhongxin. Chinese guidelines for the diagnosis and treatment of adult insomnia (2017 Edition) [J]. Chinese Journal of Neurology, 2018,51 (05): 324-335.

4. Ruyak, P.S., Bilsbury, C.D. and Malgorzata, R.A. (2004) Survey of Insomnia Treatment at Canadian Sleep Centres: Is There a Role for Clinical Psychologists? Canadian Psychology, 45, 165173. https://doi.org/10.1037/h0086983

5. Reddy, M.S. and Arindam, C. (2011) Comorbid Insomnia. Indian Journal of Psychological Medicine, 33, 1-4. https://doi.org/10.4103/0253-7176.85388

6. Nicolaides NC, Vgontzas AN, Kritikou I, Chrousos G. HPA Axis and Sleep. 2020 Nov 24. In: Feingold KR, Anawalt B, Boyce A, Chrousos G, de Herder WW, Dungan K, Grossman A, Hershman JM, Hofland J, Kaltsas G, Koch C, Kopp P, Korbonits M, McLachlan R, Morley JE, New M, Purnell J, Singer F, Stratakis CA, Trence DL, Wilson DP, editors. Endotext [Internet]. South Dartmouth (MA): MDText.com, Inc.; 2000-. PMID: 25905298. 
7. Si Y, Wang L, Lan J, Li H, Guo T, Chen X, Dong C, Ouyang Z, Chen SQ. Lilium davidii extract alleviates $\mathrm{p}$-chlorophenylalanine-induced insomnia in rats through modification of the hypothalamicrelated neurotransmitters, melatonin and homeostasis of the hypothalamic-pituitary-adrenal axis. Pharm Biol. 2020 Dec;58(1):915-924. doi: 10.1080/13880209.2020.1812674. PMID: 32924742; PMCID: PMC7534321.

8. Dujardin S, Pijpers A, Pevernagie D. Prescription Drugs Used in Insomnia. Sleep Med Clin. 2020 Jun;15(2):133-145. doi: 10.1016/j.jsmc.2020.02.002. PMID: 32386689.

9. Kalmbach DA, Cheng P, Arnedt JT, Anderson JR, Roth T, Fellman-Couture C, Williams RA, Drake CL. Treating insomnia improves depression, maladaptive thinking, and hyperarousal in postmenopausal women: comparing cognitive-behavioral therapy for insomnia (CBTI), sleep restriction therapy, and sleep hygiene education. Sleep Med. 2019 Mar;55:124-134. doi: 10.1016/j.sleep.2018.11.019. Epub 2018 Dec 28. PMID: 30785053; PMCID: PMC6503531.

10. Li Ran, Liu Jinglu, song Hongli, Xin Yaran, Li Haiyan, Zhang Yanxia, Zhao chenjing. Clinical efficacy of wrist ankle acupuncture in patients with insomnia [J]. Hebei Journal of traditional Chinese medicine, 2019,34 (03): 42-44

11. Song Yuan, Li Hong, Zhao canghuan, Wu Xianlin, Ou Shiyi. Treatment of 48 cases of insomnia with wrist ankle acupuncture [J]. Chinese Journal of Gerontology, 2014,34 (18): 5233-5234

12. Wu Wenyuan, Wei Jing, Tao Ming, on behalf of the anxiety disorder collaborative group of psychiatric branch of Chinese Medical Association. Expert consensus on diagnosis and treatment of anxiety and depression in general hospitals [J]. Chinese medical journal, 2012 (31): 2174-2181

13. Eldridge SM, Chan CL, Campbell MJ, Bond CM, Hopewell S, Thabane L, et al. CONSORT 2010 statement: extension to randomised pilot and feasibility trials. Bmj. 2016;355:i5239

14. Atalay H. Comorbidity of insomnia detected by the Pittsburgh sleep quality index with anxiety, depression and personality disorders. Isr J Psychiatry Relat Sci. 2011;48(1):54-9. PMID: 21572244.

15. Standardized manipulations of acupuncture and moxibustion-Part 19:Wrist-ankle acupuncture.

\section{Figures}




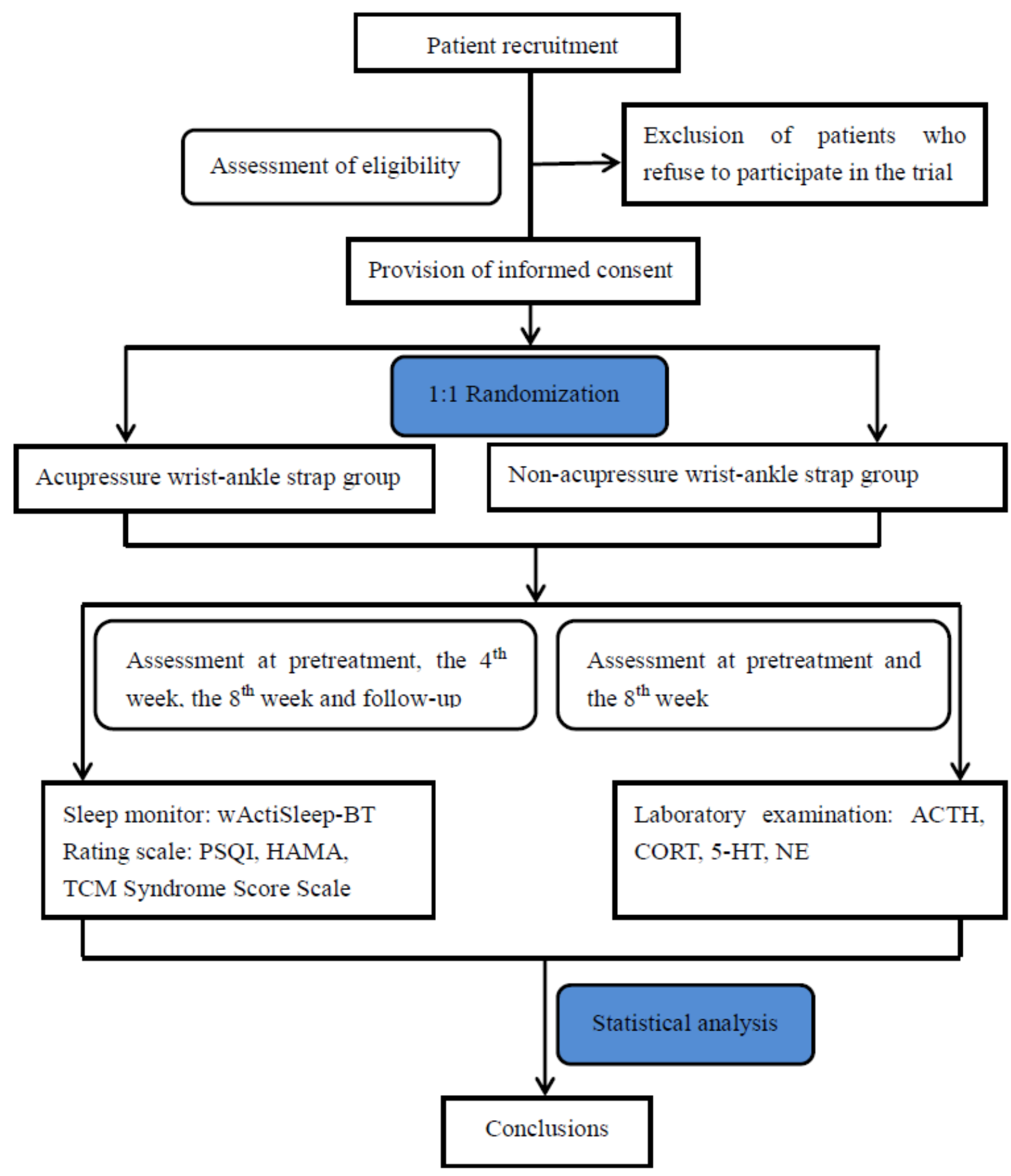

Figure 1

Flowchart of the trial. 


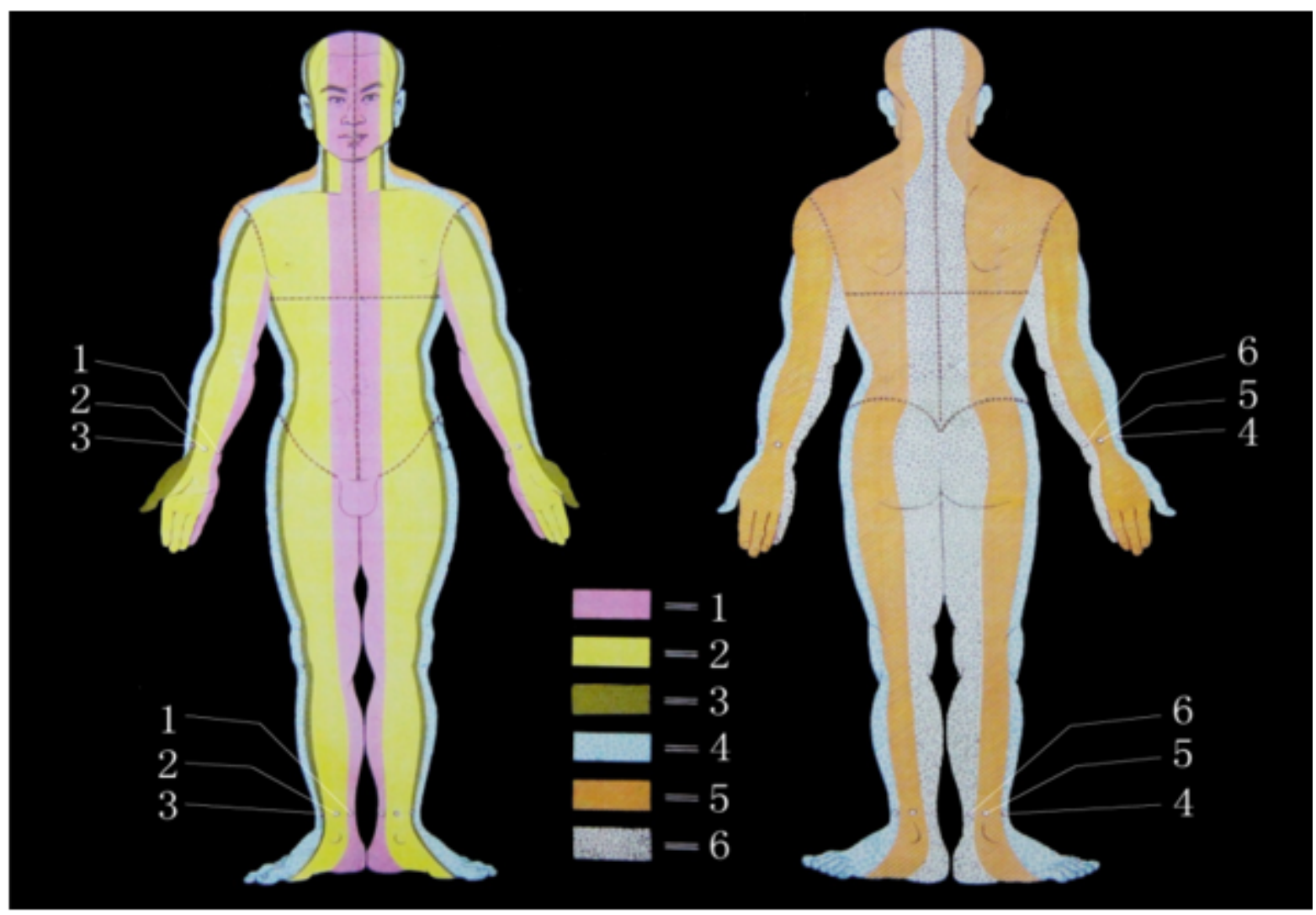

Figure 2

Wrist-ankle acupuncture (WAA) zones and needling points. 


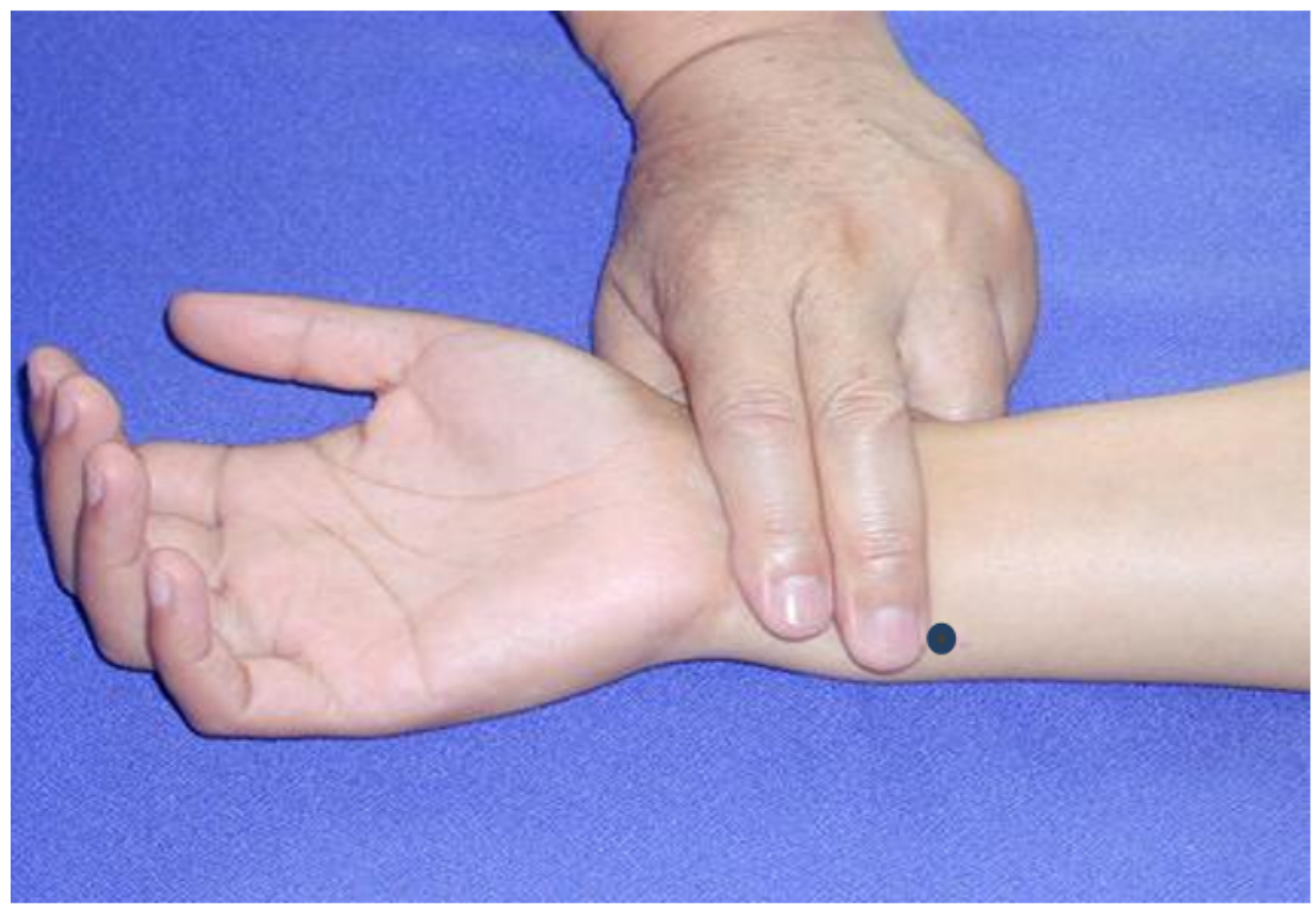

\section{Figure 3}

Location of the upper 1 point of Wrist-ankle acupuncture (WAA).
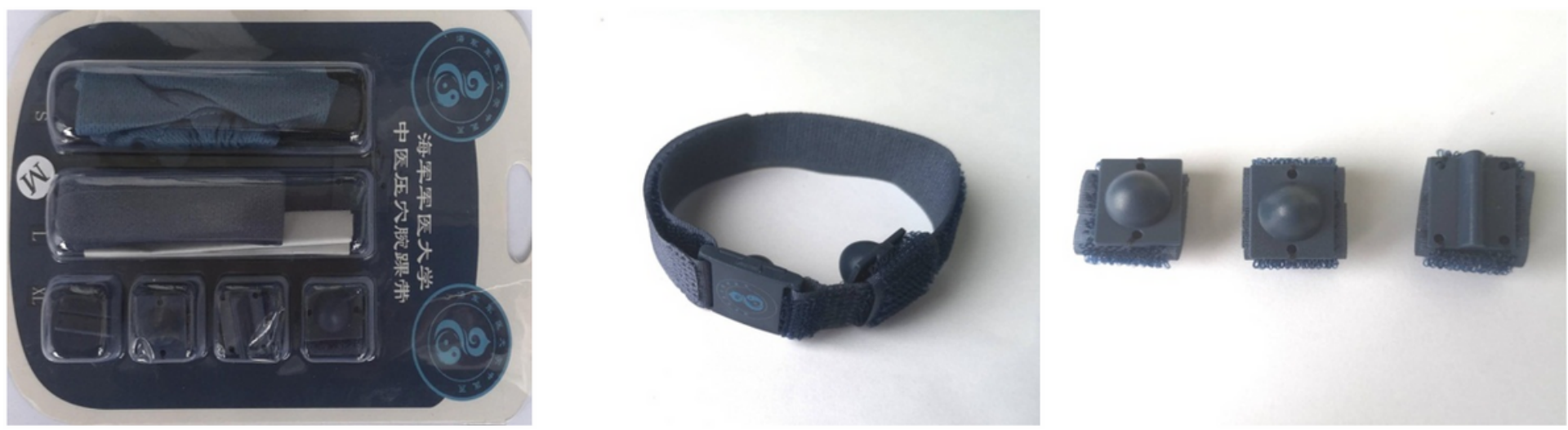

Figure 4

The acupressure wrist-ankle strap and it's accessories. 


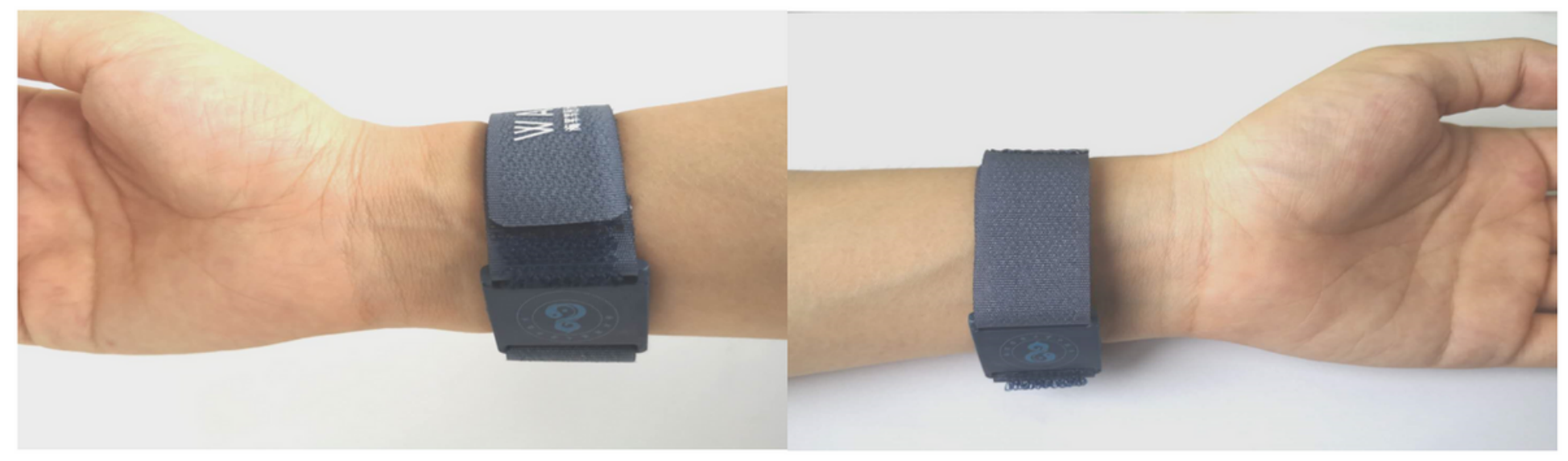

\section{Figure 5}

The acupressure wrist-ankle straps on bilateral upper 1.
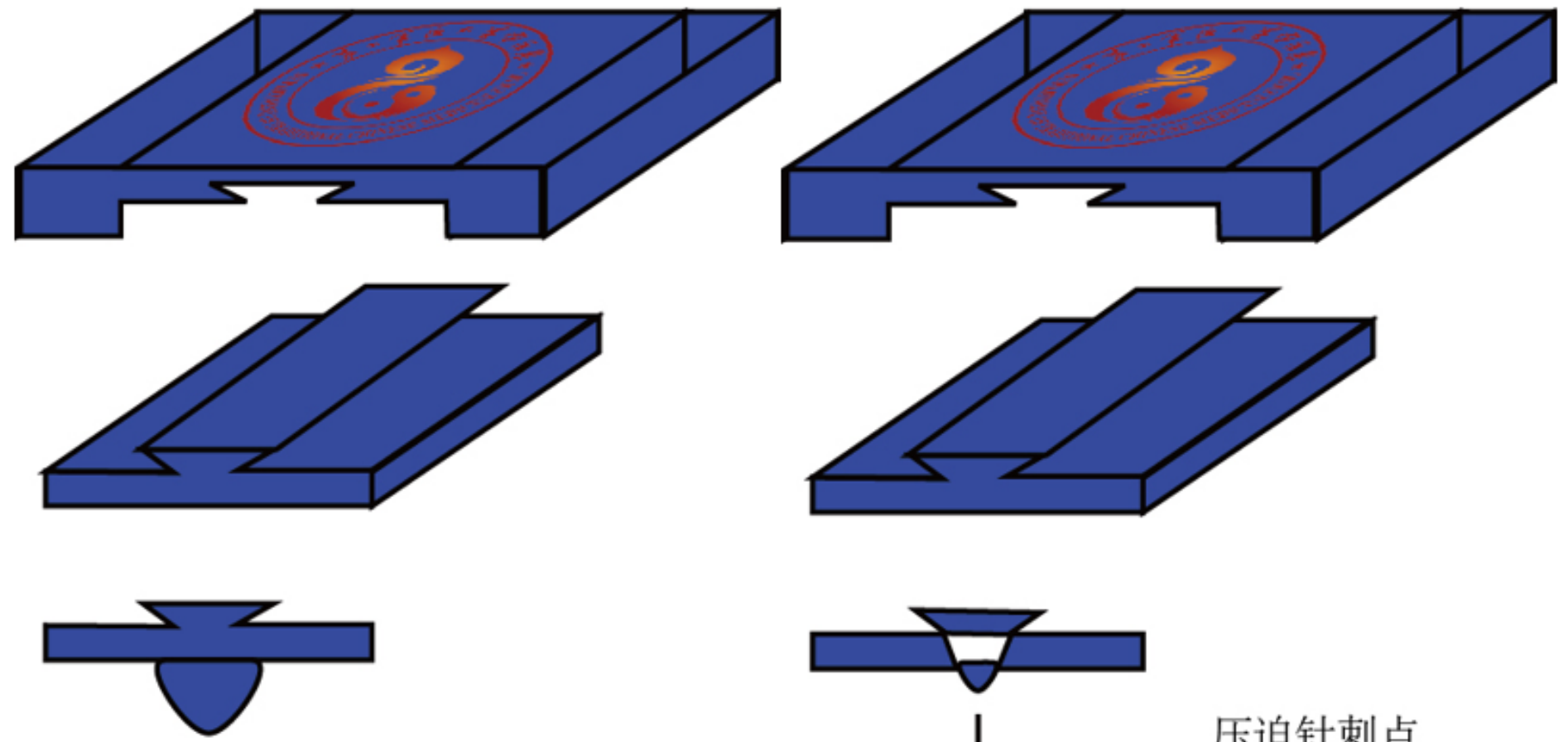

压迫针刺点， 凸起部位不收缩

A
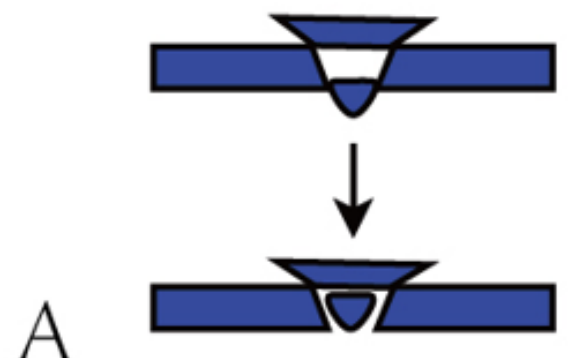

压迫针刺点, 凸起部位收缩

$\mathrm{B}$

\section{Figure 6}

The acupressure wrist-ankle strap compression device is shown in $A$, the non-acupressure wrist-ankle strap compression device is shown in B. 


\begin{tabular}{|c|c|c|c|c|c|}
\hline & \multicolumn{5}{|c|}{ STUDY PERIOD } \\
\hline \multirow[b]{2}{*}{ TIMEPOINT } & $\begin{array}{l}\text { Enrol } \\
\text { ment }\end{array}$ & Allocation & \multicolumn{3}{|c|}{ Post-allocation } \\
\hline & 招募 & 0 & 4 weeks & 8 weeks & Follow-up \\
\hline \multicolumn{6}{|l|}{ ENROLMENT: } \\
\hline \multirow{3}{*}{$\begin{array}{r}\text { Eligibility screen } \\
\text { Informed conset } \\
\text { Allocation }\end{array}$} & $\times$ & & & & \\
\hline & $\times$ & & & & \\
\hline & & $\times$ & & & \\
\hline \multicolumn{6}{|l|}{ INTERVENTIONS: } \\
\hline \multicolumn{6}{|l|}{ ankle strap group } \\
\hline \multicolumn{6}{|l|}{$\begin{array}{r}\text { Non-acupressure wrist-ankle strap } \\
\text { group }\end{array}$} \\
\hline \multicolumn{6}{|l|}{ ASSESSMENTS: } \\
\hline \multirow{2}{*}{$\begin{array}{l}\text { PSQI } \\
\text { HAMA }\end{array}$} & & $\times$ & $x$ & $x$ & $x$ \\
\hline & & $\times$ & $x$ & $\times$ & $x$ \\
\hline TCM Syndrome Score Scale & & $\times$ & $x$ & $\times$ & $x$ \\
\hline \multirow[t]{2}{*}{ wActisleep-BT } & & $x$ & $x$ & $\times$ & $x$ \\
\hline & & $x$ & & $x$ & \\
\hline ACTH & & $\times$ & & $x$ & \\
\hline CORT & & $x$ & & $x$ & \\
\hline $5-\mathrm{HT}$ & & $\times$ & & $x$ & \\
\hline
\end{tabular}

Figure 7

The schedule of enrolments, allocation, and assessments.

\section{Supplementary Files}

This is a list of supplementary files associated with this preprint. Click to download.

- SPIRITchecklistYY.docx 